Pacific

Journal of

Mathematics

SYMPLECTIC SUBMANIFOLDS FROM SURFACE FIBRATIONS

IVAN SMITH

Volume $198 \quad$ No. 1

March 2001 


\title{
SYMPLECTIC SUBMANIFOLDS FROM SURFACE FIBRATIONS
}

\author{
IVAN SMITH
}

\begin{abstract}
We give a simple construction yielding homology classes in (non-simply-connected) symplectic four-manifolds which admit infinitely many pairwise non-isotopic symplectic representatives. Examples are constructed in which the symplectic curves can have arbitrarily large genus. The examples are built from surface bundles over surfaces and involve only elementary techniques. As a corollary we see that a blow-up of any simply-connected complex projective surface contains a connected symplectic surface not isotopic to any complex curve.
\end{abstract}

\section{Symplectic tori.}

The existence of symplectic submanifolds realising certain homology classes has had a significant impact on our understanding of symplectic topology, particularly in dimension four. This raises the natural question as to the uniqueness of symplectic representatives for homology classes in instances when they exist at all. In complex geometry the uniqueness (or finiteness) of complex representatives for (co)homology classes is well-known; for instance if $X$ is a projective surface then an element $\alpha$ of $H_{2}(X)$ is dual to a cohomology class $\alpha^{*}$, and complex representatives of $\alpha$ correspond to transverse holomorphic sections of one of finitely many line bundles with first Chern class $\alpha^{*}$. (If the surface is simply connected the line bundle is unique.) These sections are points of certain projective spaces $\mathbb{P} H^{0}\left(X, L_{\alpha^{*}}\right)$; since the locus of non-transverse sections in each such space is a complex subvariety, its complement is connected, and there are at most finitely many isotopy classes of submanifold. Our main result shows that this cannot be true in the symplectic category:

\section{Theorem 1.1.}

- For every odd genus $g \neq 3$ there is a symplectic four-manifold $X$ and a homology class $C \in H_{2}(X, \mathbb{Z})$ such that $C$ may be represented by infinitely many pairwise non-isotopic connected symplectic surfaces $\left\{S_{a}\right\}_{a \in \mathbb{Z}}$ of genus $g$ inside $X$. 
- For every odd $g \neq 3$ there is a simply-connected projective surface $Z_{g}$ which admits a connected symplectic surface of genus $g$ not isotopic to any complex curve. Indeed, some blow-up of any simply connected projective surface contains a symplectic non-complex curve.

In fact it shall follow from the proof that there is no homeomorphism of pairs $\left(X, S_{a}\right) \equiv\left(X, S_{b}\right)$ where $S_{a}, S_{b}$ are a distinct pair of such symplectic surfaces. More precisely we shall show:

Proposition 1.2. The homology class $2 m\left[\Sigma_{g}\right]$ in the four-manifold $\Sigma_{g} \times \mathbb{S}^{2}$ (for $m \geq 2$ an integer and any $g \geq 1$ ) can be represented by infinitely many pairwise non-isotopic connected symplectic surfaces, each of which has genus $r$ where

$$
2-2 r=2 m(2-2 g) .
$$

The restriction to curves of odd genus arises since our construction involves branched covers of even degree; it could be removed with the obvious adaptation. Similarly one can consider twisted sphere bundles. The first instance of the proposition contains all the essential ideas and is an immediate corollary of work of Geiges [5]. For any diffeomorphism $f$ of a surface $\Sigma$ we write $Y_{f}$ for the mapping torus of $f$; in particular for $A \in S L_{2}(\mathbb{Z})$ we have $Y_{A}=\mathbb{T}^{2} \times{ }_{A} \mathbb{S}^{1}$. Geiges notes that:

- If $A_{1}, A_{2} \in S L_{2}(\mathbb{Z})$ satisfy $\left|\operatorname{tr}\left(A_{i}\right)\right|>3$ then the torus bundles $\mathbb{S}^{1} \times Y_{A_{i}}$ are diffeomorphic if and only if $A_{1}=A_{2}$.

- For any four-manifold $Z=\mathbb{S}^{1} \times Y_{A}$ as above, and given any cohomology class $a \in H^{2}(Z)$ with $a^{2}>0$ we can find an integral symplectic form representing the class $a$. Moreover if $a(F)>0$ where $F$ is a torus fibre, we can assume the symplectic structure is compatible with the fibration.

- None of the four-manifolds $Z_{A}$ above admit any complex structure.

Now the mapping class group $S L_{2}(\mathbb{Z})$ for the torus differs only by a central $\pm I$ from the braid group for four points in the sphere $\mathbb{S}^{2}$. It follows that given any matrix $A \in S L_{2}$ we can define a braid on four strands; when the image of the braid is connected we may view this as a map $\beta_{A}: \mathbb{S}^{1} \rightarrow \mathbb{S}^{1} \times \mathbb{S}^{2}$ with image $\Gamma_{\beta}$. (If the braid is not connected the domain of $\beta_{A}$ will be some disjoint union of circles, and we use the same notation for the image.)

Lemma 1.3. For any such braid, the product $\mathbb{S}^{1} \times \Gamma_{\beta} \subset \mathbb{S}^{1} \times\left(\mathbb{S}^{1} \times \mathbb{S}^{2}\right)$ is a symplectic submanifold of $\mathbb{T}^{2} \times \mathbb{S}^{2}$ with its standard product symplectic form.

Proof. This is a trivial computation. With co-ordinates $(\theta, \phi),(u, v)$ on $\mathbb{T}^{2} \times \mathbb{S}^{2}$ the symplectic form locally has the shape $d \theta \wedge d \phi+d u \wedge d v$ whilst the tangent space at any point to the submanifold is spanned (in a suitable normalisation) by $\partial / \partial \theta$ and $\partial / \partial \phi+\alpha \partial / \partial u+\gamma \partial / \partial v$ for some $\alpha, \gamma$ not both zero. The only non-zero term arises from the $\theta, \phi$ component of the symplectic form and this is always positive. 
For any braid $\beta_{A}$ we have built a symplectic submanifold in the homology class 4 [Torus] $\subset \mathbb{T}^{2} \times \mathbb{S}^{2}$. It is obviously possible to choose an infinite family of matrices $A_{i}$ for which the corresponding braids $\beta_{A_{i}}$ are connected and the matrices have trace $\left|\operatorname{tr} A_{i}\right|>3$. We claim that all of the associated symplectic submanifolds are non-isotopic (indeed not equivalent under any diffeomorphism of pairs). For given any such submanifold, we can form the double cover of $\mathbb{T}^{2} \times \mathbb{S}^{2}$ branched over this class, and the result is precisely the torus bundle $\mathbb{S}^{1} \times Y_{A}$. Since all of these manifolds are non-complex, but the branched cover of a complex manifold over a complex divisor does admit a complex structure, the submanifolds are not isotopic to complex curves. Again, since the $\mathbb{S}^{1} \times Y_{A}$ represent infinitely many diffeomorphism types, so we must have infinitely many isotopy classes of branch locus. The genus formula given in (1.2) holds since symplectic submanifolds satisfy the adjunction formula. This establishes the proposition in the case $m=2, g=$ 1. The second part of (1.1) for these special values is now immediate.

Corollary 1.4. Let $\mathcal{E}_{n}$ denote the $n$-th fibre sum of the rational elliptic surface with itself. Then $\mathcal{E}_{n}$ contains a connected symplectic surface not isotopic to any complex curve.

Proof. The $\mathcal{E}_{n}$ are all elliptically fibred, and we may take a trivial fibre sum with $\mathbb{T}^{2} \times \mathbb{S}^{2}$ to exhibit a connected symplectic torus representing the homology class $4[$ Fibre]. On the other hand, the (unique) complex representative for this homology class is a disjoint union of four parallel fibres, which is clearly not isotopic to any connected curve.

For $n=1,2$ this gives connected symplectic non-complex submanifolds in $\mathbb{C P}^{2} \sharp 9 \overline{\mathbb{C P}}^{2}$ and $K 3$. A conjecture due to Siebert and Tian asserts that there are no such symplectic surfaces in minimal rational ruled manifolds, and the example serves to demonstrate the necessity of the minimality assumption.

Via the same method of fibre summation, and taking higher $m$ in (1.2) one obtains a homology class on a simply connected complex surface with $N$ distinct symplectic representatives for any required $N$, distinguished by connectivity. However, Fintushel and Stern [4] have observed that for any braid $\beta_{A}$ if we include the associated symplectic submanifold $\mathbb{S}^{1} \times \Gamma_{\beta_{A}}$ into an elliptic surface $E$ for which $\pi_{1}(E \backslash\{$ Fibre $\})=0$ using this fibre sum trick, then the fundamental group of the complement is cyclic, independent of $A$. Hence we cannot use the same naive algebraic topological methods to give infinite families of representatives. With more sophistication, taking branched covers and using the Seiberg-Witten invariants, Fintushel and Stern have nonetheless shown that the above submanifolds do remain pairwise non-isotopic inside $\mathcal{E}_{i}$. Their methods of computation do not apply, unfortunately, to distinguish curves of higher genus. 


\section{Generalisations.}

In this section we will extend the previous discussion to cover the other cases of (1.2), in particular proving (1.1). The first step is to move from the homology class 4 [Fibre] inside $\mathbb{T}^{2} \times \mathbb{S}^{2}$ to $2 m$ [Fibre]. For this we just need an analogue of Geiges' result: Then we can work with branched covers and the submanifolds $\mathbb{S}^{1} \times \Gamma_{\beta}$ for $\beta$ a braid corresponding to a hyperelliptic mapping class at some genus $h$, and the above proof will hold. (In particular precisely the same proof that the submanifolds are symplectic will apply.) It is straightforward to adapt Geiges' proof. Since we have used the notation $\Gamma_{\beta}$ to denote a graph above, we will write $\operatorname{Out}\left(\pi_{1}\left(\Sigma_{g}\right)\right)$ to denote the mapping class group of a genus $g$ surface, typically denoted $\Gamma_{g}$.

\section{Proposition 2.1.}

Let $g \geq 1$ and $\gamma \in \operatorname{Out}\left(\pi_{1}\left(\Sigma_{g}\right)\right)$ be such that:

- $\gamma$ is not periodic,

- 1 is not an eigenvalue of the action on cohomology $\gamma_{*}: H^{1}\left(\Sigma_{g}\right) \rightarrow$ $H^{1}\left(\Sigma_{g}\right)$.

Then the manifold $X_{\gamma}=\mathbb{S}^{1} \times\left(\mathbb{S}^{1} \times_{\gamma} \Sigma_{g}\right)$ is a symplectic fibration with no compatible Kähler metric. Indeed the manifold has no complex structure at all.

Proof.

- The manifolds we are considering are smooth fibrations of surfaces over tori; since base and fibre are aspherical, it follows that so is the total space. Thus

$$
H_{*}\left(\Sigma_{g} \tilde{\times} \mathbb{T}^{2} ; \mathbb{Z}\right)=H_{*}\left(\pi_{1}\left(\Sigma_{g} \tilde{\times} \mathbb{T}^{2}\right) ; \mathbb{Z}\right)
$$

where we define the group homology in the usual way ${ }^{1}[\mathbf{3}]$. There is an extension of groups

$$
0 \rightarrow \pi_{1}\left(\Sigma_{g}\right) \rightarrow \mathcal{G} \rightarrow \mathbb{Z}^{2} \rightarrow 0
$$

where $\mathbb{Z}^{2}$ acts on $\pi_{1}\left(\Sigma_{g}\right)$ via the monodromies $\gamma_{i} \in \operatorname{Out}\left(\pi_{1}\left(\Sigma_{g}\right)\right)$, and $\mathcal{G}=\pi_{1}(X)$. We will later assume one monodromy is trivial (so the fourmanifold is $\mathbb{S}^{1}$ times a mapping torus). In any case, there is a HochschildSerre spectral sequence - the group homology analogue of the Leray-Serre spectral sequence, valid because of the asphericity - for which

$$
E_{p, q}^{2}=H_{p}\left(\mathbb{Z}^{2} ; H_{q}\left(\pi_{1} \Sigma_{2}\right)\right) \Longrightarrow H_{p+q}(\mathcal{G} ; \mathbb{Z})=H_{p+q}\left(X_{\gamma_{1}, \gamma_{2}} ; \mathbb{Z}\right)
$$

The arguments of Thurston [8] show the total space of the fibration is symplectic iff $E_{0,2}^{\infty}=\mathbb{Z}=E_{0,2}^{2}$.

Riemann surfaces are Eilenberg-Maclane spaces so

$$
H_{*}\left(\pi_{1} \Sigma_{g}\right)=H_{1}\left(\Sigma_{g}\right)=\mathbb{Z}^{2 g} .
$$

\footnotetext{
${ }^{1}$ That is, for a group $\Gamma$ take a projective resolution $\mathcal{R}_{\Gamma}$ of $\mathbb{Z}$ over $\mathbb{Z}[\Gamma]$ and define $H_{*}(\Gamma ; M)$ to be the homology of the sequence $\mathcal{R} \otimes_{\Gamma} M$.
} 
We get the following free (projective!) resolution:

$$
0 \rightarrow \mathbb{Z}^{2 g} \stackrel{\theta_{*}}{\longrightarrow} \mathbb{Z}^{4 g} \stackrel{\phi_{*}}{\longrightarrow} \mathbb{Z}^{2 g}
$$

where the maps are defined by

$$
\begin{gathered}
\theta_{*}:\left(\begin{array}{l}
x \\
y
\end{array}\right) \mapsto\left(\begin{array}{c}
{\left[\left(\gamma_{1}\right)_{*}-I\right]\left(\begin{array}{l}
x \\
y
\end{array}\right)} \\
{\left[I-\left(\gamma_{2}\right)_{*}\right]\left(\begin{array}{l}
x \\
y
\end{array}\right)}
\end{array}\right) \\
\phi_{*}:\left(\left(\begin{array}{l}
x \\
y
\end{array}\right),\left(\begin{array}{l}
z \\
t
\end{array}\right)\right) \mapsto\left[\left(\gamma_{1}\right)_{*}-I\right]\left(\begin{array}{l}
x \\
y
\end{array}\right)+\left[\left(\gamma_{2}\right)_{*}-I\right]\left(\begin{array}{l}
z \\
t
\end{array}\right) .
\end{gathered}
$$

From this we see that $\operatorname{ker} \phi_{*} / \operatorname{im} \theta_{*}=E_{1,1}^{2}$ and since $d_{2}: E_{1,1}^{2} \rightarrow E_{-1,2}^{2}=0$ we have $E_{1,1}^{2}=E_{1,1}^{\infty}$. Also $E_{0,2}^{2}=\mathbb{Z}$ for $H_{2}\left(\pi_{1}\right)$ is a trivial module. Since

$$
H_{2}=E_{0,2}^{\infty}+E_{1,1}^{\infty}+E_{2,0}^{\infty}
$$

we see that if $\operatorname{rank}\left(E_{1,1}^{2}\right)=b_{2}-2$ then there is a compatible symplectic structure. To find $b_{i}(X)$ we make the assumption that the first monodromy $\gamma_{1}$ is trivial; for then the $E^{2}$-page of the spectral sequence for the 3-manifold $Y_{\gamma}=S^{1} \times_{\gamma} \Sigma_{g}$ is trivial with all differentials zero. It follows from the analogous free resolution in the 3 -dimensional case that

$$
H_{1}\left(Y_{\gamma}\right)=\mathbb{Z} \oplus \operatorname{ker}\left(\gamma_{*}-\mathrm{id}: \mathbb{Z}^{2 g} \rightarrow \mathbb{Z}^{2 g}\right)
$$

and in particular, if $1 \notin$ Eigenvalues $\left.\gamma_{*}\right\}$ then $b_{1}\left(X_{\gamma}\right)=b_{1}\left(Y_{\gamma}\right)+1=2$.

But in this case, $b_{2}(X)=2$ and $\operatorname{rank}\left[E_{1,1}^{2}\right]=0 \Leftrightarrow$ symplectic structures exist. Equivalently, we require (2.3) to have rank zero when $\left(\gamma_{2}\right)_{*}=$ id. But the complex has $H_{1}$ term with rank equal to $\left(2 \cdot \operatorname{rank}\left[\operatorname{ker}\left(\gamma_{*}-I\right)\right]\right)$, giving the result.

To see there are no possible complex structures, when $g>1$ one can most easily resort to the classification of surfaces, and check that these manifolds are not on the list; for they have $c_{2}=0$ despite being minimal, and have $b_{1}=2$ so cannot be Kodaira surfaces or minimal surfaces of class VII. The $g=1$ case (already used in the first section) was covered by Geiges' work.

Remark 2.4. A few words on notation. By $\Gamma_{g}^{\text {hyp }}$ we shall denote the hyperelliptic mapping class group; if we fix a hyperelliptic Riemann surface $\Sigma_{g}$ then there is a distinguished hyperelliptic involution $\iota \in \operatorname{Out}\left(\pi_{1}\left(\Sigma_{g}\right)\right)$ inside the mapping class group of $\Sigma_{g}$, and $\Gamma_{g}^{\text {hyp }}$ denotes the set of elements of the mapping class group commuting with $\iota$. We also have the familiar groups $\Gamma_{0}^{2 g+2}$ and $\operatorname{Br}_{2 g+2}\left(\mathbb{S}^{2}\right)$ - the mapping class group of the marked sphere and the braid group on $(2 g+2)$-strands in the sphere, respectively [2]. There are isomorphisms (for instance from explicit presentations)

$$
\Gamma_{0}^{2 g+2} \sim \Gamma_{g}^{\text {hyp }} /\langle\iota\rangle \sim \operatorname{Br}_{2 g+2}\left(\mathbb{S}^{2}\right) /\langle\Delta\rangle
$$


where $\Delta$ generates the centre $Z\left(\operatorname{Br}_{2 g+2}\left(\mathbb{S}^{2}\right)\right) \cong \mathbb{Z}_{2}$. In particular, given any braid in the sphere, we have a hyperelliptic mapping class defined uniquely up to composition with the involution $\iota$, and all mapping classes arise this way. (Indeed given a mapping class we can build a braid inside $\mathbb{S}^{2} \times I$ and the central $\mathbb{Z}_{2}$-ambiguity corresponds to the different possible twistings of the sphere bundle over the circle $\mathbb{S}^{1}$ obtained by closing the ends of the interval I.) With these delicacies understood, we shall pass freely between braids and mapping classes henceforth; required choices can be made arbitrarily by the reader.

It is easy to obtain in this way infinitely many diffeomorphism types of symplectic non-complex surface bundle over a torus. Indeed, for different monodromies in general the homeomorphism types will differ, distinguished by the fundamental group. If we choose the non-trivial monodromy to be hyperelliptic then we can associate to the surface bundle a braid (on $2 g+2$ strands, where the fibre has genus $g$ ) such that the surface bundle is the double cover of $\mathbb{T}^{2} \times \mathbb{S}^{2}$ over the surface $\mathbb{S}^{1} \times \Gamma_{\beta}$ as before. For connected braids, this surface is a symplectic torus in the homology class $(2 g+2)$ [Fibre]. The genus one case of the Proposition (1.2) follows.

To obtain symplectic surfaces of higher genus we use the fibre sum operation. Recall that given two symplectic fibrations $Z_{1} \rightarrow B_{1}, Z_{2} \rightarrow B_{2}$ with diffeomorphic fibre, then after scaling the symplectic forms to give the fibres equal area we may glue tubular neighbourhoods of fixed fibres to obtain a new fibration $Z_{1} \sharp_{F} Z_{2} \rightarrow B_{1} \sharp B_{2}$ which covers the (usual) connected sum of the base surfaces. One of the choices in this construction is of a twisting diffeomorphism of the fibre with which we make the identification. In particular, given any two hyperelliptic genus $g$ surface bundles over a torus, we may take a fibre sum (twisted by an element of the hyperelliptic mapping class group) to produce a hyperelliptic surface fibration by genus $g$ surfaces over a genus two base; this new fibration still admits a symplectic structure compatible with the fibration.

Now think of our two surface bundles $Z_{i}$ as branched covers of $\mathbb{T}^{2} \times \mathbb{S}^{2} \rightarrow \mathbb{T}^{2}$ over symplectic torus multi-sections. We may fibre sum the two sphere bundles along a sphere fibre. Moreover, by the relative form of Gompf's surgery, once we fix a fibre in each $Z_{i}$ we distinguish a set of $2 g+2$ points in the fibre which are the intersection points with the symplectic branch curve. View the hyperelliptic diffeomorphism as (induced by) an element of the braid group for $2 g+2$ points on the sphere and choose a lift of this homotopy class of diffeomorphisms to a diffeomorphism fixing the marked points. We can glue the two sphere bundles with a twist by this diffeomorphism and Gompf's theorems [6] provide a symplectic structure on the new sphere bundle over $\Sigma_{2}$ with respect to which the two tori glue to give a symplectic surface of genus $2 g+3$. This is an unramified cover of the base $\Sigma_{2}$ of degree 
$2 g+2$. As we vary the original torus bundles and hyperelliptic gluing map, we obtain infinite families of symplectic fibrations over a genus two curve which we can view as branched covers of sphere bundles over connected symplectic surfaces.

Lemma 2.5. The above techniques yield infinite families of symplectic noncomplex curves of any genus $2 g+3$ with $g \geq 1$ inside $\Sigma_{2} \times \mathbb{S}^{2}$.

Proof. From the above, we need show first that the symplectic curves are not complex, and secondly that they are not pairwise isotopic; it will therefore be sufficient to show that the hyperelliptic surface bundles we construct represent infinitely many non-complex diffeomorphism types. Indeed once we know we have infinitely many diffeomorphism types the statement on complex structures will follow: Since a smooth complex fibre bundle will be determined by a representation $\pi_{1}$ (Base) $\rightarrow$ Aut(Fibre) where Aut denotes the holomorphic automorphism group. For curves of genus $g>1$ this is finite, and there can only be finitely many such automorphisms; for curves of genus one, the fibre bundles have $c_{2}=0$ and the classification of complex surfaces applies (over a genus two base we will have $b_{1} \geq 4$ and hence they cannot be minimal surfaces of class VII).

We are reduced to the homeomorphism classification of hyperelliptic surface bundles. Since base and fibre are again aspherical, the topology is completely encoded in the fundamental group; in the homotopy exact sequence

$$
0 \rightarrow \pi_{1} \text { (Fibre) } \rightarrow \pi_{1}(Z) \rightarrow \pi_{1}\left(\Sigma_{2}\right) \rightarrow 0
$$

the extension is determined by the monodromies which can be viewed as relations amongst generators for $\pi_{1}(Z)$. Indeed the bundle is given by a representation $\pi_{1}\left(\Sigma_{2}\right) \rightarrow \Gamma_{g}^{\text {hyp }}$ into the hyperelliptic genus $g$ mapping class group. Given any two hyperelliptic mapping classes $\phi, \psi$ and a presentation

$$
\pi_{1}\left(\Sigma_{2}\right)=\langle a, b, c, d \mid[a, b][c, d]=1\rangle
$$

we may take $a \mapsto \phi, b \mapsto 1, c \mapsto q^{-1} \psi q, d \mapsto 1$ to obtain a representation as above. Here $q$ is an arbitrary element of the hyperelliptic mapping class group, and as it varies we can obtain infinitely many different conjugacy classes of representation (since there are infinitely many conjugacy classes of element in $\Gamma_{g}^{\text {hyp }}$ ). It then follows from the homotopy exact sequence that we can obtain infinitely many distinct fundamental groups for the total space. The result follows.

To complete the proof of (1.1) observe that there is a simply connected projective surface with a Lefschetz fibration of genus 2 curves - for instance blow up the $K 3$ given by double covering $\mathbb{P}^{2}$ over a sextic at the two preimages of the basepoint of a pencil of lines. We can fibre sum $\Sigma_{2} \times \mathbb{S}^{2}$ into this genus two fibration, carrying with us a symplectic surface of arbitrarily large odd 
genus from the above construction (choosing the braid to obtain the trivial sphere bundle $\Sigma_{2} \times \mathbb{S}^{2}$ and not some twisted bundle). The symplectic surface represents a homology class $2 m$ [Fibre] for some $m$ and the unique complex representative of this fibre is again disconnected. The result of the fibre summation remains simply connected since in $K 3 \sharp 2 \mathbb{\mathbb { C P }}^{2}$ the fundamental group of the complement of a fibre is trivial. Now for any complex projective surface $Z$ we may find a Lefschetz pencil of curves of some large genus. Blowing up the base-points of this pencil we have a Lefschetz fibration, inside which we can identify a trivial fibration $\Sigma_{r} \times D$ for a complex disc $D \subset \mathbb{P}^{1}$. Iterating the fibre sum construction above, we can find a symplectic curve of any large odd genus inside $\Sigma_{r} \times \mathbb{S}^{2}$ which is disjoint from some fibre $\mathbb{S}^{2}$ and hence can be assumed to lie in the disc bundle $\Sigma_{r} \times D \subset Z$. The usual arguments show this is not the complex representative of the relevant homology class $2 m$ [Fibre] (which is unique when the surface is 1-connected). This completes the proof.

Remark 2.6. In complex geometry one is often as interested in nodal and cuspidal curves as smooth curves; for instance these appear as branch loci of generic projections of projective surfaces to $\mathbb{C P}^{2}$. Following Auroux's work on symplectic four-manifolds [1] the symplectic geometry of these surfaces is now of interest. Moishezon [7] gave examples of nodal cuspidal curves in $\mathbb{C P}^{2}$ which were not isotopic to complex nodal cuspidal curves; all of his examples contained cusps. The surfaces we construct in $\mathbb{T}^{2} \times \mathbb{S}^{2}$ can be pushed down to $\mathbb{S}^{2} \times \mathbb{S}^{2}$ where they acquire nodes (but not worse singularities). For careful braids (for instance of several components) the resulting surfaces can be made symplectic. It is not clear if this is of interest, however, since the symplectic surfaces built in this way appear always to have nodes of both positive and negative intersection.

Acknowledgements. The examples of the first section appeared in my D.Phil thesis and I am grateful to Simon Donaldson for general inspiration. Minor errors were kindly pointed out by Bernd Siebert and Ron Fintushel before I prepared the later drafts of the work, and it is a pleasure to acknowledge their contribution here.

\section{References}

[1] D. Auroux, Symplectic four-manifolds as branched coverings over $\mathbb{C P}^{2}$, Invent. Math., 139 (2000), 551-602.

[2] J. Birman, Braids, Links and Mapping Class Groups, Princeton University Press, 1975.

[3] K.S. Brown, Cohomology of Groups, Springer, 1982.

[4] R. Fintushel and R. Stern, Symplectic surfaces in a fixed homology class, J. Diff. Geom., 52 (1999), 203-222. 
[5] H. Geiges, Symplectic structures on $\mathbb{T}^{2}$ bundles over $\mathbb{T}^{2}$, Duke Math. J., 67 (1992), 539-55.

[6] R. Gompf, A new construction of symplectic manifolds, Annals of Math., 142 (1995), 527-595.

[7] B.G. Moishezon, Stable branch curves and braid monodromies, Algebraic Geometry, Chicago 1980, LNM, 862, Springer, (1981), 107-192.

[8] W. Thurston, Some simple examples of symplectic manifolds, Proc. Amer. Math. Soc., 55 (1976), 467-8.

Received May 11, 1999 and revised October 5, 1999.

New College

OXFORD OX1 3BN

ENGLAND

E-mail address: smithi@maths.ox.ac.uk 